\title{
The role of social and psychological factors in attitudes towards personalised nutrition
}

\author{
K.H Hart, S. Nabbijo and S. Wilson-Barnes \\ School of Biosciences \& Medicine, Faculty of Health \& Medical Sciences, University of Surrey, GU2 7XH, UK
}

Personalised nutrition is a novel public health strategy aimed at facilitating and sustaining positive diet and lifestyle changes. Providing diet and lifestyle advice (often via a mobile application) that is more biologically relevant (tailored to an individual's genetic, physiological and/or microbiome profile) is anticipated to be more effective and also more engaging and motivating for the user

${ }^{(1,2)}$. However, the extent to which personalisation can override demographic mediators of eating patterns and behaviour change, such as income or gender, and the individual differences in response to personal health data remain to be seen. Furthermore, the utilisation of modern mobile and wireless technologies to support personalised health objectives (often referred to as $\mathrm{m}$-health) should also be appraised.

This study aimed to investigate the impact of sociodemographic and psychological factors on self-reported behaviour change and attitudes towards m-health delivered personalised nutrition in a sample of UK adults, and to characterise those most likely to engage with personalised nutrition.

An online survey was developed and distributed via Qualtrics (version 2019; Provo UT) to gather data on participant characteristics, self-reported physical and mental health and attitudes towards health and nutrition, behaviour change and personalised nutrition. The University of Surrey Ethics Committee gave a favourable ethical opinion and the survey was open to anyone over 18 years.

The sample $(\mathrm{n}=204)$ were predominantly female $(87 \%)$, aged $18-24$ years $(69 \%)$ and of white ethnic background $(78 \%)$ with $96 \%$ having A level qualifications or above. Men were more likely to perceive that general dietary advice lacked specificity $(60 \%$ agreement versus $35 \%$ for women, $p=0.02$ ). However overall, cost and time were more commonly reported barriers to engaging with generalised lifestyle and nutrition advice. Conversely, $65 \%$ of respondents reported that they would follow personalised nutrition advice, and this was more common in those who were younger and who reported greater health consciousness $(p \leq 0.01)$. Interestingly, fewer respondents agreed that they would follow advice based on their genetic (56\% agreement) or microbiome data (50\%) specifically. Issues of data safety and the number of daily prompts and notifications they would receive were highlighted by survey respondents as potential negative aspects of personalised nutrition delivered by $\mathrm{m}$-health. The time commitment required to engage with $\mathrm{m}$-health was cited as another possible barrier (47\%) yet almost half of participants agreed personalised $\mathrm{m}$-health could facilitate goal setting and the implementation of lifestyle changes.

Whilst new technology and personalised nutrition, individually or in combination, have the potential to revolutionise public health, offering economic, social and physiological benefits, this study suggests that engagement cannot be assumed. Engagement may be influenced by demographic and psycho-social factors such as age, gender and health consciousness and future research should identify how to promote engagement with these approaches to maximise their impact.

\section{References}

1. JM, Ferguson LR, Tai ES \& Mathers JC (2018) BMJ 361.

2. Mathers JC (2019) EFSA Journal, 17 (S1), 1-9. 